\title{
Review of: "Revealing Genetic Diversity, Population Structure and Cultivar-Specific SSR Alleles in Pistachio Using SSR Markers"
}

\author{
Xiao-Bin Liu ${ }^{1}$ \\ 1 Kunming Institute of Botany, Chinese Academy of Sciences
}

Potential competing interests: The author(s) declared that no potential competing interests exist.

The authors developed 92 polymorphic SSR markers in the economically important species Pistacia vera. They provided the polymorphism information of each mark and revealed the correlation between the geographical origin and the genetic background of the cultivars.

This is an important and meaningful work to pave the road for further germplasm resources studies in the future. Regarding the amount of the SSR marker the author developed and the multiple origins of the cultivars, the reviewer suggests digging the data deeper to tell a more interesting story.

The reviewer's suggestions are detailed below:

1 Could you compare the genetic diversity of the cultivars from different origins? And try to discuss the correlation between the genetic diversity pattern to the local industry. The potential hypothesis is the more developed industry might be lead to the lower genetic diversity of the germplasm. Even you can compare the genetic diversity of the wild type vs. cultivars.

2 Is that possible to trace back the breeding history of the cultivars from the STRUCTURE analysis? Because we can observe some hybridization occurred in some cultivars from Figure2

3 Could you find your markers that can be linked to some agronomic traits of your cultivars. If you can find this kind of marker, that should be very important for further breeding work and raise the value of this paper.

4 Since you have 66 cultivars that come from different countries, so if you can preliminary construct the "core collection" of Pistacia vera based on the genetic diversity data, you can raise the value of this paper again. You can find an example in building a core collection from here 
https://link.springer.com/article/10.1186/s41065-017-0038-0

The reviewer believes if the authors dig their data deeper, and organize a more interesting story, they can publish their work on Genetic Resources and Crop Evolution. 